\title{
Non-Hermitian Kibble-Zurek Mechanism with Tunable Complexity in Single-Photon Interferometry
}

\author{
Lei Xiao, ${ }^{1}$ Dengke Qu, ${ }^{2,1}$ Kunkun Wang, ${ }^{1}$ Hao-Wei Li, ${ }^{3}$ Jin-Yu Dai, ${ }^{3}$ Balázs Dóra $\odot,{ }^{4}$ Markus Heyl, ${ }^{5}$ \\ Roderich Moessner, ${ }^{5}$ Wei Yi, ${ }^{3,6}$ and Peng Xue ${ }^{1, *}$ \\ ${ }^{1}$ Beijing Computational Science Research Center, Beijing 100084, China \\ ${ }^{2}$ Department of Physics, Southeast University, Nanjing 211189, China \\ ${ }^{3}$ CAS Key Laboratory of Quantum Information, University of Science and Technology of China, Hefei 230026, \\ China \\ ${ }^{4}$ Department of Theoretical Physics and MTA-BME Lendület Topology and Correlation Research Group, \\ Budapest University of Technology and Economics, Budapest 1521, Hungary \\ ${ }^{5}$ Max-Planck-Institut für Physik komplexer Systeme, Dresden 01187, Germany \\ ${ }^{6}$ CAS Center For Excellence in Quantum Information and Quantum Physics, Hefei 230026, China
}

(Received 3 December 2020; accepted 5 April 2021; published 30 April 2021)

\begin{abstract}
Non-Hermitian descriptions of quantum matter have seen impressive progress recently, with major advances in understanding central aspects such as their topological properties or the physics of exceptional points, the non-Hermitian counterpart of critical points. Here, we use single-photon interferometry to reconstruct the non-Hermitian Kibble-Zurek mechanism and its distinct scaling behavior for exceptional points, by simulating the defect production upon performing slow parameter ramps. We report progress along two axes. First, we realize parameter ramps across higher-order exceptional points, providing experimental access to their theoretically predicted characteristic Kibble-Zurek scaling behavior in the defect production. Second, we extend the scaling properties to the defect fluctuations, which enables us to extract the correlation length and dynamical exponents of the underlying the exceptional point (EP) and therefore to gain direct experimental information about the universality class of the EPs. Our work represents a step toward increasing the experimental complexity of non-Hermitian quantum time evolution, as part of the quest to move the frontier from single-particle physics toward increasingly complex settings.
\end{abstract}

DOI: 10.1103/PRXQuantum.2.020313

\section{INTRODUCTION}

The foundational axioms of quantum mechanics impose a Hermitian structure on Hamiltonians. However, it is now appreciated that rich and unconventional phenomena can arise in settings where the constraints enforced by such Hermitian structures are absent. This happens rather generically for systems in touch with an environment; experimental instances occurring in photonics $[1,4]$, cold atoms $[5,9]$, mechanical systems $[10,12]$, and electric circuits [13, 17] have revealed rich single-particle properties induced by non-Hermiticity. In particular, the so-called exceptional points (EPs) [18,22] provide the non-Hermitian [23,27] counterpart to the familiar critical points [28] in Hermitian

\footnotetext{
*gnep.eux@gmail.com

Published by the American Physical Society under the terms of the Creative Commons Attribution 4.0 International license. Further distribution of this work must maintain attribution to the author(s) and the published article's title, journal citation, and DOI.
}

band structures [29], [30], leading to critical phenomena unique to non-Hermitian systems. Whereas most previous studies focus on single-particle properties, we are now faced with the challenge of extending their experimental reach, by realizing specific phenomena characteristic of nonunitary dynamics on the one hand and allowing the study of more complex problems on the other, shifting the frontier toward the many-body realm.

In this work, we report progress along both axes using single-photon interferometric networks as an experimental platform to simulate many-body dynamics driven by non-Hermitian Hamiltonians. The nonunitary many-body dynamics is broken down into the time evolution of different quasiparticle modes, which are then encoded in the polarizations of photons, detectable through single-photon interference. Such a framework enables the engineering of highly tunable non-Hermitian band structures and allows us to realize different classes of EPs with varying complexity. Mathematically, at an EP, two (or more) complex eigenvalues and eigenstates coalesce [18]. These eigenstates then no longer form a complete basis, which in turn 
feeds into distinct properties of the EP compared to those at Hermitian critical points. We consider various types of non-Hermitian EPs, culminating in a higher-order EP, the counterpart of the familiar higher-order critical point, which generically appears in multidimensional phase diagrams upon tuning a pair of parameters. Such a framework enables us to systematically characterize the novel nonunitary dynamics pertaining to different types of EPs, which is specifically visible in the dynamics of defect generation upon passage through the exceptional and/or critical point in the time domain, as captured by the venerable Kibble-Zurek mechanism. This theory was originally proposed for domain formation in the early universe [31], applied to superfluid helium [32], [33], and extended to dissipative systems [34]. Experimentally, the theory has been confirmed using optical interferometers [35], superconducting qubits [36], Bose-Einstein condensates [37], self-organized rubidium atoms [38], and Rydberg atoms [39]. We simulate such ramps in a non-Hermitian manybody setting and probe the characteristic universal scaling behavior of the resulting defect density:

$$
n \sim v^{d^{*} v /(z v+1)} .
$$

Here, $v$ denotes the sweep velocity and $v$ the correlation length exponent. Remarkably, the defect density $n$ obeys a scaling form even in the non-Hermitian case with, however, one crucial difference. While for conventional critical points $d^{*}=d$ is identical to the spatial dimension $d$, in the non-Hermitian setting the effective dimension [19] $d^{*}=d+z$ contains a shift by the dynamical critical exponent $z$. We go beyond the conventional Kibble-Zurek consideration and generalize its scaling behavior to defect fluctuations, i.e., noise [40]. Our theory predicts that $\Delta n^{2}$ obeys similar scaling with $d^{*}=d$ for the Hermitian case, while $d^{*}=d+2 z$ for non-Hermitian dynamics. These quantities are all accessible in the photon interferometric networks. Our measurements of $n$ and $\Delta n^{2}$ confirm a power-law dependence, with exponents aligning with the Kibble-Zurek prediction for both Hermitian and nonHermitian cases. For the non-Hermitian case, we take one crucial step further by recognizing that the modified exponents for $n$ and $\Delta n^{2}$ put us in a position to extract from the experimental data even the individual exponents $v$ and $z$, thereby gaining direct information about the universality class.

With the ability to tune the band structure complexity, we then also address the Kibble-Zurek mechanism for higher-order EPs and determine their characteristic properties experimentally. Our approach is based on simulating sets of independent modes, mimicking the dynamics of effectively noninteracting quasiparticles upon crossing critical and exceptional points. This technique provides a flexible framework for realizing and probing nonunitary dynamics with high tunability and control, with the potential to push the frontier further toward the quantum simulation of many-body effects.

\section{MODEL, OBSERVABLES, AND PROTOCOL}

We consider translationally invariant quantum systems that can be described in terms of independent modes:

$$
H=\sum_{p} H_{p}, H_{p}=p \sigma_{x}+\Delta \sigma_{y}+i \Gamma \sigma_{z}
$$

Here, $H_{p}$ denotes a $2 \times 2$ matrix represented in terms of Pauli operators $\sigma_{\alpha}(\alpha=x, y, z)$ and parametrized through the momentum $p$ of the mode and the couplings $\Delta, \Gamma$. This Hamiltonian exhibits a genuine non-Hermitian character due to the complex mass term, with $\Gamma \in \mathbb{R}$ implying $H^{\dagger} \neq$ $H$. The resulting spectrum $E_{ \pm}(p)= \pm \sqrt{p^{2}+\Delta^{2}-\Gamma^{2}}$ is gapless at $p^{2}+\Delta^{2}=\Gamma^{2}$, which marks the location of a second-order EP. It is the key goal of this work to experimentally study the dynamical consequences of such EPs [41] and to contrast with those of conventional Hermitian critical points [28], [39].

The model in Eq. (2) is popular in a variety of nonHermitian settings. It can be regarded as the continuum limit of the famous Hermitian Su-Schrieffer-Heeger model, with $p$ the momentum and $\Delta$ the alternating hopping, supplemented by alternating imaginary, non-Hermitian on-site energies [42] $\Gamma$, which is also equivalent to a quantum Ising chain in an imaginary transverse field. Similar nonHermitian Hamiltonians can also be realized by optical waveguides [2], [43], microcavities [44], or electric circuits [13]. By using a time-dependent $\Delta$ and $\Gamma$, a variety of relevant protocols can be realized.

Based on the general protocol that we outline below, the nonunitary dynamics can be further enriched by engineering $H_{p}$ as an enlarged $4 \times 4$ matrix, enabling us to access a mode structure of increased complexity, and the concomitant unconventional higher-order EPs.

For our protocol, we initialize each mode $p$ in its ground state $\left|\Psi_{p}\right\rangle$ with $\Gamma=0$. At time $t=0$, we then start the nonequilibrium process by linearly increasing $\Gamma \propto t$, driving the system through either a critical or an exceptional point. The defects in the final state $\left|\Psi_{p}(\tau)\right\rangle$ at time $t=\tau$ are quantified via

$$
\sigma_{z}(p, \tau)=\frac{\left\langle\Psi_{p}(\tau)\left|\sigma_{z}\right| \Psi_{p}(\tau)\right\rangle}{\left\langle\Psi_{p}(\tau) \mid \Psi_{p}(\tau)\right\rangle} .
$$

Since the Hamiltonian encompasses $2 \times 2$ Pauli matrices, its temporal dynamics can be solved exactly, similar to the Hermitian Landau-Zener problem. However, since we are mostly interested in the defect production at the end of the time evolution, it is more illuminating and straightforward to borrow powerful scaling ideas from conventional Hermitian critical points and apply them to 
the non-Hermitian case. Such a practice predicts [19] that the defect density follows a universal scaling form in terms of the corresponding critical exponents, as

$$
\sigma_{z}(p, \tau)-\sigma_{z}^{\mathrm{eq}}(p)=\frac{1}{\tau^{z v /(z v+1)}} f\left(p^{z} \tau^{z v /(z v+1)}\right),
$$

where $f(x)$ is a scaling function characteristic of the chosen protocol and $\sigma_{z}^{\mathrm{eq}}(p)$ denotes the equilibrium or steadystate value. The prefactor $\tau^{-z v /(z v+1)}$ on the right-hand side is only present for an EP and arises due to the fact that the defect state can decay back to the ground state in the nonHermitian case, as discussed in Ref. [19]. This, together with the fractional critical exponents $z$ and $v$, represents the major difference in comparison to the Hermitian case.

While the exact solution of the time-dependent Schrödinger equation would also yield $f(x)$, its explicit form is not needed to obtain the universal scaling of the defect density; it would only determine nonuniversal prefactors, which is not the focus of this investigation. The total defect density $n=(2 \pi)^{-1} \int d p \sigma_{z}(p, \tau)$ according to the Kibble-Zurek prediction Eq. (1) shows universal behavior, when measured with respect to its equilibrium or steady-state value, $n_{\text {eq }}$ (see the Supplemental Material [45]). For ease of notation, we set the velocity of $p$ modes and $\hbar$ to unity and the density of states to $1 / \pi$.

\section{EXPERIMENTAL IMPLEMENTATION}

Experimentally, we realize for each mode $p$ the time-evolution operator of the targeted $H_{p}$ in singlephoton interferometry. The basis states are encoded in the horizontal and vertical polarizations of single photons, with $|H\rangle=(1,0)^{T}$ and $|V\rangle=(0,1)^{T}$. The photons are initialized in the ground state $\left|\Psi_{p}(0)\right\rangle$ of $H_{p}$ with $\Gamma=0$ through a series of wave plates with $p$-dependent parameters (see the Supplemental Material [45]) and are sent to the interferometric network as illustrated in Fig. 1. The associated time-evolution operator $U_{p}(\tau)$ is realized by three successive operations $U_{p}(\tau)=R\left(\theta_{2}, \varphi_{2}, \vartheta_{2}\right) L\left(\phi_{H}, \phi_{V}\right) R\left(\theta_{1}, \varphi_{1}, \vartheta_{1}\right)$. Here, the rotation operators $R\left(\theta_{j}, \varphi_{j}, \vartheta_{j}\right)(j=1,2)$ are experimentally implemented using a set of sandwich-type wave plates involving two quarter-wave plates (QWPs) and a HWP $[Q(\theta)-H(\varphi)-Q(\vartheta)]$, each with three independent parameters (setting the angles of the wave plates, $\theta, \varphi$, and $\vartheta)$. The polarization-dependent loss operator $L\left(\phi_{H}, \phi_{V}\right)$ contributes the nonunitary dynamics and is experimentally achieved by a Mach-Zehnder interferometer. After entering the interferometer at a beam displacer (BD), photons are separated in a polarization-dependent fashion and traverse the interferometer in a superposition of the upper and lower paths. Finally, the photons interfere at the second BD. Photons with horizontal (the number of photons proportional to $\left|\sin 2 \phi_{H}\right|^{2}$ ) and vertical polarizations $\left(\left|\sin 2 \phi_{V}\right|^{2}\right)$ are kept for the next operation,

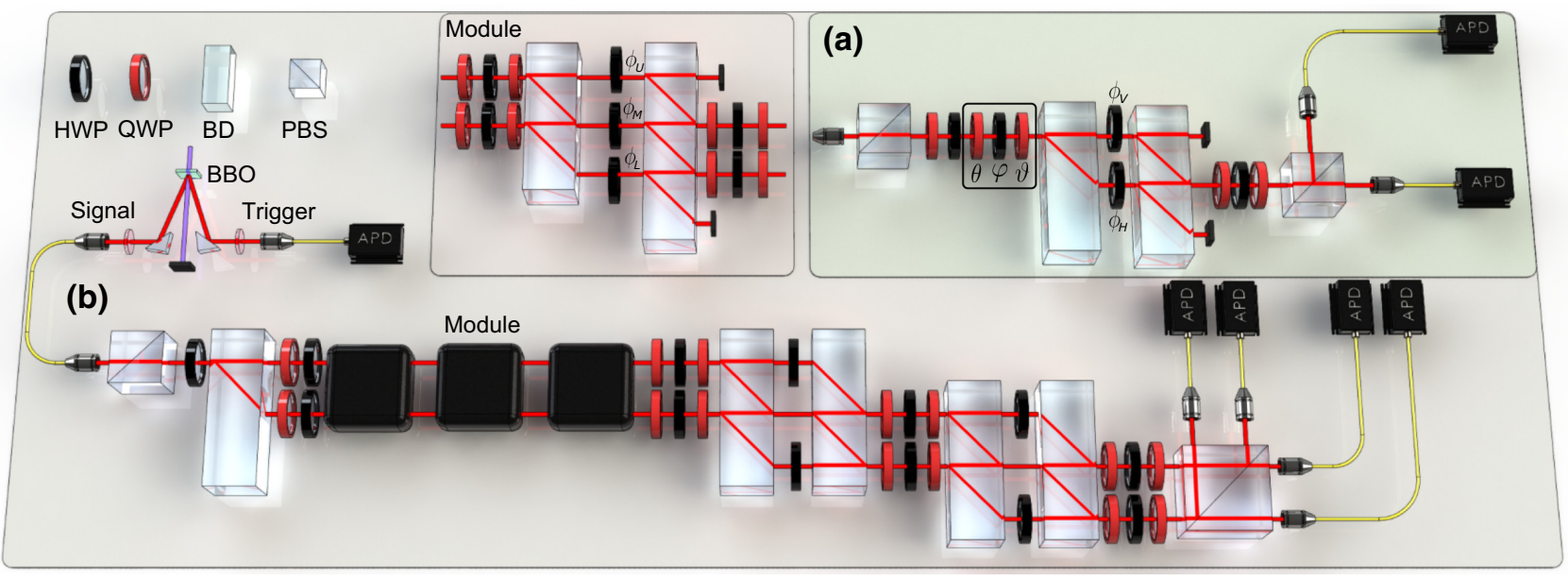

FIG. 1. The experimental setup. As a first step, a photon pair is created via spontaneous parametric down-conversion (SPDC). One of the photons serves as a trigger, while the other is projected into the polarization state $|H\rangle$ or $|V\rangle$ with a polarizing beam splitter (PBS) and a half-wave plate (HWP) before entering the interferometric network. (a) The experimental setup for the nonunitary dynamics of a single-qubit parity-time- $(\mathcal{P} \mathcal{T})$ symmetric system. (b) The experimental setup for the nonunitary dynamics of a four-level system, where the qudit is encoded in polarization and spatial degrees of freedom. To prepare the initial state, heralded single photons pass through a PBS and a HWP with tailored setting angles and are split by a birefringent calcite beam displacer (BD) into two parallel spatial modes: upper and lower modes. After passing through wave plates inserted into the optical paths of the two spatial modes, the photons are prepared in the initial state $\left|\Psi_{p}(0)\right\rangle$ or $\left|\varphi_{p}\right\rangle$ (see the main text for definitions), through $p$-dependent wave plates. The nonunitary evolution is then realized by three modules involving BDs and wave plates and projective measurements are performed by a cascaded interferometer and avalanche photodiodes (APDs). 
while the others are lost from the optical setup. Two HWPs, with setting angles $\phi_{H}$ and $\phi_{V}$, respectively, are inserted into the two arms of the interferometer to tune the parameters of the loss operator. We choose the parameters $\left\{\theta_{j}, \varphi_{j}, \vartheta_{j}, \phi_{H}, \phi_{V}\right\}$ such that the sequence of three operations reproduces the targeted $U_{p}(\tau)$. Such a scheme enables us to implement any desired nonunitary operator for two-level systems [30], with each given set of parameters $p, \Delta, \Gamma$ of the Hamiltonian in Eq. (2) determined by a set of setting angles according to our analytical decomposition protocol (see the Supplemental Material [45]). We measure $\sigma_{z}(p, \tau)$ by recording the relative photon counts in the basis $\{|H\rangle,|V\rangle\}$ through a PBS and avalanche photodiodes (APDs), with a typical peak count of 160000 photons. In order to estimate the total defect density $n$, we utilize Gauss-Legendre quadrature, which uniquely determines the specific $p$ points once the integration domain is fixed.

\section{HERMITIAN KIBBLE-ZUREK SCALING}

We first validate the setup by reproducing the wellknown Kibble-Zurek scaling for Hermitian quantum critical points with quantitative accuracy. Concretely, we consider $\Delta=0$ and $\Gamma=-i \Delta_{0} t / \tau$, yielding a mass term that increases linearly with time. Figure 2(a) shows the results for $\sigma_{z}(p, \tau)$ in scaled units so as to achieve a data collapse with the predicted exponents: the resulting total defect density $n$ [see Fig. 2(c)] is consistent with power-law behavior over more than one decade. From a fit $n-n_{\text {eq }} \sim \tau^{-\alpha}$, we obtain $\alpha=0.494(2)$, which, within the error bars, agrees with the theoretical prediction $\alpha=0.5$ for the underlying equilibrium quantum critical point of Ising universality class with $d=z=v=1$. Here, the constant $n_{\mathrm{eq}}$ denotes the expected adiabatic value for $\sigma_{z}(p, \tau)$ in the $\tau \rightarrow \infty$ limit.

\section{NON-HERMITIAN KIBBLE-ZUREK SCALING}

For the central aspect of this work, the non-Hermitian Kibble-Zurek scaling at EPs, we consider two cases: first, an exact $\mathcal{P} \mathcal{T}$-symmetric ramp with $\Delta=\Delta_{0}$ and $\Gamma=$ $\Delta_{0} t / \tau$ [see Figs. 2(b) and 2(c)], where the energy spectrum $E_{ \pm}(p)$ of the system remains real throughout the ramp approaching an EP at $t=\tau$; and, second, a fully non-Hermitian drive with $\Delta=0, \Gamma=\Gamma_{0} t / \tau$ [see Fig. 3]. To reduce experimental error for the fully non-Hermitian drive, we sample the mode-resolved defect densities and analyse the scaling behavior in regions $p \gtrless \Gamma_{0}$ separately. Whereas the difference in the integrated densities between the two regions gives the total integrated density as before, the smallness of such a difference would lead to small photon counts and significantly larger error bars. By analysing the two regions separately, as we show below, a universal scaling behavior is established, since both regions respect the same scaling law.

For both cases, the data collapse across the varying ramp times $\tau$, characteristic of the Kibble-Zurek mechanism but now realized for non-Hermitian systems. The resulting scaling functions, however, differ markedly from the Hermitian case in Fig. 2(a). In particular, the full nonHermitian drive provides a manifestly non-Hermitian feature: the double-peak structure in Fig. 3(a) implies modes $p$ with lower occupation than for the purely adiabatic limit, which is impossible for the Hermitian case [46,48].

While the scaling functions appear to have a rather unconventional form, the integrated total defect density $n$ robustly exhibits power-law behavior, as illustrated in Figs. 2(c) and 3(b). The associated exponents follow the modified scaling law in Eq. (1), with the effective dimension $d^{*}$. For the exact $\mathcal{P} \mathcal{T}$ ramp, the fit $n-n_{\mathrm{eq}} \sim \tau^{-\alpha}$ yields an exponent $\alpha=0.68(1)$, consistent with the theoretical prediction $\alpha=2 / 3$ for the underlying EP with (a)

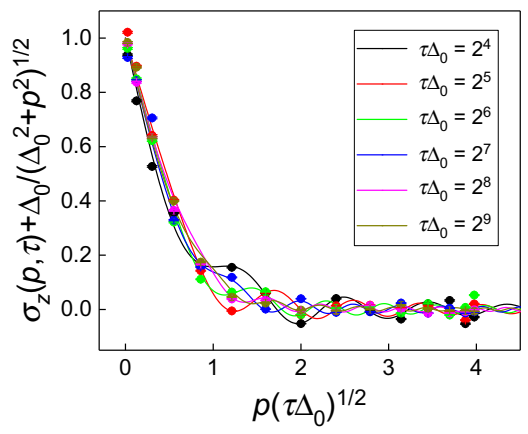

(b)

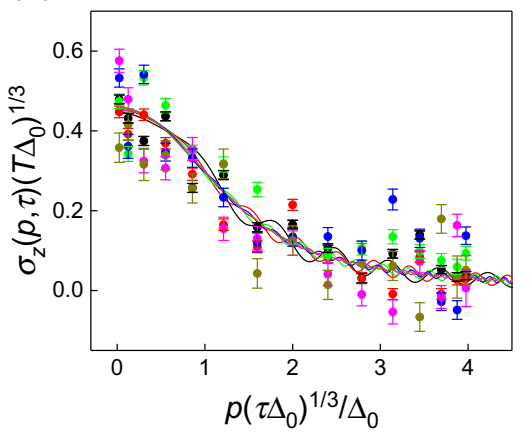

(c)

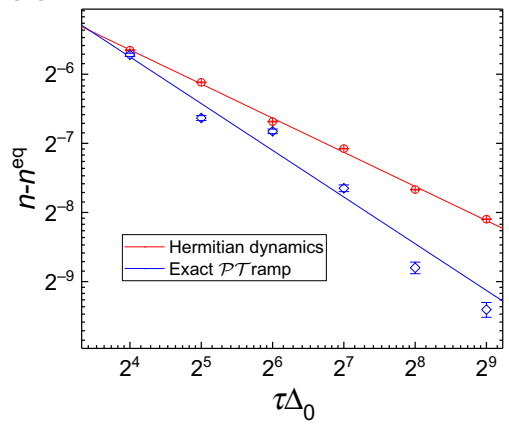

FIG. 2. The Kibble-Zurek scaling of defect production for parametric ramps in Hermitian and non-Hermitian exact $\mathcal{P} \mathcal{T}$ systems. The data collapse for the momentum-resolved defect density for (a) the Hermitian case and (b) the exact $\mathcal{P} \mathcal{T}$ ramp. The dots with error bars display the experimental measurements and solid lines refer to numerical simulations. (c) The total defect density $n$ relative to the adiabatic values $n_{\mathrm{eq}}$ for the two considered cases as a function of the ramp time $\tau$. The solid lines show the results for the best powerlaw fit $n-n_{\mathrm{eq}} \sim \tau^{-\alpha}$ to the experimental data. The fitted exponents $\alpha=0.494(2)$ for the Hermitian scaling (red) and $\alpha=0.68(1)$ for the exact $\mathcal{P} \mathcal{T}$ ramp (blue). 
(a)

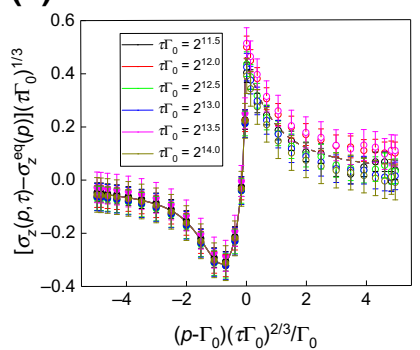

(b)

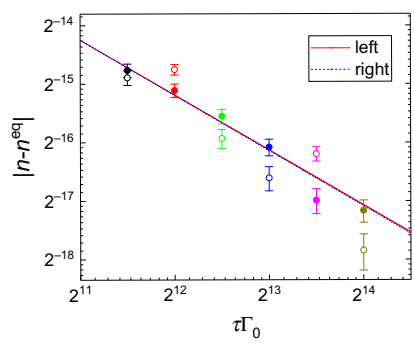

FIG. 3. The Kibble-Zurek scaling of defect production for parameter ramps across non-Hermitian EPs. (a) The data collapse for the momentum-resolved defect density for the full non-Hermitian drive. (b) The total defect density relative to the adiabatic values as a function of the ramp time $\tau$. As discussed in the main text, we sample the left $\left(p<\Gamma_{0}\right)$ and right $\left(p>\Gamma_{0}\right)$ regions separately. The red solid (black dashed) line in (b) shows the results for the best power-law fits $\left|n-n_{\mathrm{eq}}\right| \sim \tau^{-\alpha}$ for the left (right) region, with the fitted exponents $\alpha=0.94(7)$ in both regions.

critical exponents $d=z=1, v=1 / 2$. For the full nonHermitian drive, the fitted exponents are both $\alpha=0.94(7)$ for the two regions with $p \gtrless \Gamma_{0}$ [see Fig. 3(b)]. These are in accordance with the $d=v=1$ and $z=1 / 2$ exponents, together with the power-law scaling of the total defect density from Eq. (1). We note that while other sets of critical exponents could, in principle, also yield $\alpha=1$, a unique set of critical exponents are obtained when the scaling of the defect density is combined with the scaling of defect fluctuations, as we demonstrate later. These fractional critical exponents are typical even around noninteracting exceptional points in non-Hermitian theories, while they would be rather uncommon in noninteracting Hermitian critical points. These results demonstrate the high accuracy with which our experiments can probe the dynamics of non-Hermitian systems. The increasing experimental error bars for longer evolution times in the nonunitary dynamics [Figs. 2(c) and 3(b)] are mainly due to the nonunitary photon losses: a smaller number of photons implies a larger statistical error. Importantly, the influence of imperfections appears weaker for the total defect density $n$ than for the mode-resolved one $\sigma_{z}(p, \tau)$. This happens because the errors in different modes are statistically independent and hence suppressed upon integration: the majority of experimental imperfections originate from wave plates and BDs that are independently tuned in different $p$ sectors.

\section{KIBBLE-ZUREK AT HIGHER-ORDER EXCEPTIONAL POINTS}

The key next step is to increase the complexity of the non-Hermitian Hamiltonians, accessing previously unreachable physical properties. Here, we achieve this by enlarging the mode matrix $H_{p}$ to be of $4 \times 4$ form, providing an experimental access to a higher-order EP. Here,

$$
H_{p}^{(4)}=\left(\begin{array}{cccc}
0 & \Delta-\Gamma & 0 & i p \\
\Delta+\Gamma & 0 & \Delta-\Gamma & 0 \\
0 & \Delta+\Gamma & 0 & \Delta-\Gamma \\
-i p & 0 & \Delta+\Gamma & 0
\end{array}\right) .
$$

For $\Gamma=0$, the model is Hermitian, while for $\Gamma=\Delta$, it is exact $\mathcal{P} \mathcal{T}$ symmetric and it features a fourth-order EP at $p=0$. Here, the spectrum scales as approximately $p^{1 / 4}$ due to detuning from the EP, while the gap scales as approximately $\sqrt{\Gamma-\Delta}$ at $p=0$, suggesting critical exponents $z=1 / 4$ and $z v=1 / 2$. Similar to the second-order $\mathrm{EP}$, the fractionality of dynamical critical exponents is a direct consequence of the order of the EP. Besides realizing a fourth-order EP for single-particle physics, this $4 \times 4$ Hamiltonian also represents a minimal many-body setting, i.e., a two-body system in terms of two interacting qubits (see the Supplemental Material [45]), therefore representing a first step toward developing quantum simulation and technology for interacting systems in this setting. By increasing the size of the Hamiltonian matrix, more complex many-body systems can be realized due to the high tunability of the experimental setup, in spite of the noninteracting nature of the photons. Further, since we have full experimental control over the form of the Hamiltonian that we simulate, it is possible to continuously tune the couplings between qubits, starting from a noninteracting block-diagonal form.

The four basis states are now encoded in the polarizations and spatial modes of single photons and given by $\left\{|U H\rangle=(1,0,0,0)^{T},|U V\rangle=(0,1,0,0)^{T},|D H\rangle=(0,0,1\right.$, $\left.0)^{T},|D V\rangle=(0,0,0,1)^{T}\right\}$. Here, $|U\rangle$ and $|D\rangle$ represent, respectively, the upper and lower spatial modes of the photons [see Fig. 1(b)]. The experimental implementation of the calculated $U_{p}^{(4)}$, however, is different from that of $U_{p}$ for two-level systems, which is realized exactly by BDs and wave plates. Here, instead, we approximate $U_{p}^{(4)}$ with a series of modules, each consisting of two BDs and a set of wave plates (see the Supplemental Material [45]).

We characterize the final momentum-resolved defect density through $M_{y}(p)-M_{y}^{\mathrm{eq}}(p)$, where $M_{y}(p)=\left\langle\Psi_{p}(\tau)\right.$ $\left.\left|M_{y}\right| \Psi_{p}(\tau)\right\rangle /\left\langle\Psi_{p}(\tau) \mid \Psi_{p}(\tau)\right\rangle, \quad M_{y}^{\mathrm{eq}}(p)=\left\langle\varphi_{p}\left|M_{y}\right| \varphi_{p}\right\rangle \approx$ $-\sqrt{1+\sqrt{2}}|p|^{1 / 4}$ for small $p$ to a very good approximation, with $M_{y}$ given by $M_{y}=i\left(\partial H_{p}^{(4)} / \partial \Gamma\right)$ and $\left|\varphi_{p}\right\rangle$ being the eigenstate of $H_{p}^{(4)}$ with the largest imaginary eigenvalue under the condition $\Gamma=\Delta$.

The measured $M_{y}(p)-M_{y}^{\mathrm{eq}}(p)$, for various $\tau$, again exhibits a scaling collapse [see Fig. 4(a)]. The defect production in the presence of a higher-order critical point exhibits a distinct feature, different from the previously studied cases in Figs. 2(b) and 3(a). Specifically, $M_{y}(p)-$ $M_{y}^{\mathrm{eq}}(p)$ develops a pseudogaplike feature around $p=0$ and increases monotonically with the momentum. The 
(a)

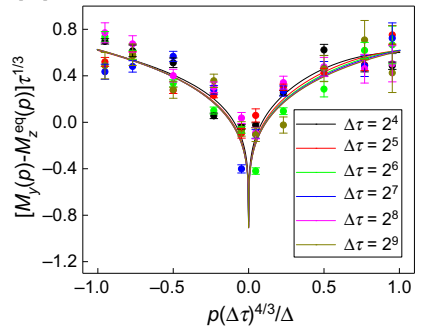

(b)

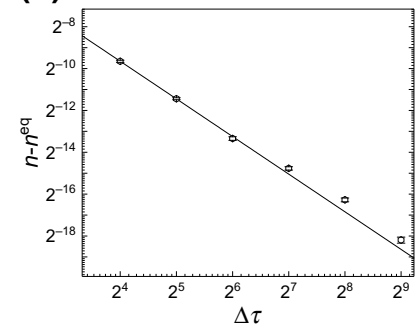

FIG. 4. The Kibble-Zurek scaling for a higher-order EP. (a) The data collapse for the momentum-resolved defect density for different ramp times $\tau$. The dots with error bars refer to the experimental measurements and the solid lines depict the exact numerical solution. (b) The total defect density as a function of the ramp time $\tau$ exhibits algebraic behavior. The solid line represents the best power-law fit to the experimental data, with the fitted exponent $\alpha=1.80$ (5). By moving further away from EP4, deviations from the ideal scaling for larger values of $p$ would appear. This can be attributed to the additional EP2s at infinity, which imply levels that are very close to each other pairwise for any finite $p$, leading to interlevel tunnelings.

total defect density $n$, as shown in Fig. 4(b), exhibits power-law behavior fitted to $\alpha=1.80(5)$, close to the Kibble-Zurek prediction, $\alpha=5 / 3$, while the error bars are larger compared to those of lower-order EPs. Note that the exact scaling relation depends on factors such as how various collapsing bands disperse from the EP or the presence of multicriticality, which become ever more abundant and complicated with the order of EP. Our experiment with a fourth-order EP thus demonstrates, on the fundamental level, the growing richness of critical phenomena as the experimental complexity is scaled up toward the many-body regime.

\section{DEFECT FLUCTUATIONS}

With our highly controllable interferometric photon network, we now take the non-Hermitian Kibble-Zurek scenario one step further by experimentally accessing quantities beyond what has been investigated before. In general, fluctuations or noise can be as important as the signal itself [40], which, for example, has been used to demonstrate the presence of fractional charge carriers for the fractional quantum Hall effect via noise measurements [49]. Here, we show that the combined knowledge of defect densities and their fluctuations opens up the possibility of gaining further experimental insights into the nature of the corresponding EPs, which would not be available from the defect density $n$ alone. While the scaling of $n$ [see Eq. (1)] provides information on a certain combination of the critical exponents $z$ and $v$, we find that the additional measurement of the defect fluctuations allows us to extract the individual exponents itself and thereby to experimentally obtain essential information about the universality class of the underlying EPs.
In the following, we consider the defect fluctuations of $n=\sum_{p} \mathcal{O}(p)$, where $\mathcal{O}(p)=\sigma_{z}(p)$ for all the considered cases except $n(p)=M_{y}(p)$ for the higher-order EP. Since the variance turns out to be always finite after the time evolution, we measure it with respect to the corresponding steady-state value in order to determine how fast nonadiabatic effects die out upon approaching the steady state, similarly to the mean, i.e., $n$. Therefore, we consider

$$
\Delta n^{2}=\left[\operatorname{Var}(n)_{\tau}-\operatorname{Var}(n)_{\mathrm{eq}}\right] / \mathcal{N},
$$

where the variance, $\operatorname{Var}(n)=\left\langle(n-\langle n\rangle)^{2}\right\rangle$, is taken with respect to the finite time evolved or the steady-state wave function, respectively, and $\mathcal{N}$ denotes the number of considered momentum states.

We find that this quantity also exhibits universal scaling (see the Supplemental Material [45]), as

$$
\Delta n^{2} \sim \tau^{-v(d+2 z) /(z v+1)} .
$$

Compared to the Hermitian case, it is crucial to note that $\Delta n^{2}$ contains an extra factor of $2 z$, which puts us in the position to individually extract $v$ and $z$. Defining the two exponents $\alpha_{n}$ and $\alpha_{\Delta}$ via $n \sim \tau_{Q}^{-\alpha_{n}}$ and $\Delta n^{2} \sim \tau_{Q}^{-\alpha_{\Delta}}$, we obtain

$$
v=\frac{\alpha_{\Delta}-2 \alpha_{n}}{d\left(\alpha_{\Delta}-\alpha_{n}-1\right)}, \quad z=d \frac{\alpha_{n}-\alpha_{\Delta}}{\alpha_{\Delta}-2 \alpha_{n}} .
$$

From the experimental data for $\Delta n^{2}$ demonstrated in Fig. 5 (see also the Supplemental Material [45]), a power-law dependence on the ramp time $\tau$ can be clearly identified. Importantly, the obtained exponents are all consistent with theoretical predictions within the error bars. While for the Hermitian dynamics, the exponents are already known [50], in the Supplemental Material [45], we derive the scaling of $\Delta n^{2}$ for the non-Hermitian cases. Measuring the strength of the defect fluctuations relative to the mean via $\delta n=\sqrt{\left|\Delta n^{2}\right|} /\left|n-n_{\mathrm{eq}}\right| \sim \tau^{\nu d /[2(z v+1)]}$ for all considered Hermitian and non-Hermitian cases, we find that these normalized fluctuations become larger for the non-Hermitian drive where $\delta n \sim \tau^{1 / 3}$ and even $\delta n \sim \tau^{2 / 3}$ for the higherorder EP, as compared to the Hermitian case where $\delta n \sim$ $\tau^{1 / 4}$. Consequently, we find that the non-Hermitian KibbleZurek mechanism not only yields modified scaling laws but also stronger fluctuations in the defect production. Based on Eq. (8), we can now estimate the critical exponents of the underlying higher-order EP, with $\alpha_{n}=1.80$ and $\alpha_{\Delta}=2.11$. This implies $v=2.16$ and $z=0.21$, which is close to the theoretically predicted values $v=2$ and $z=1 / 4$.

\section{DISCUSSION AND CONCLUSIONS}

Our work constitutes the first experimental investigation of non-Hermitian Kibble-Zurek scaling due to 
(a)

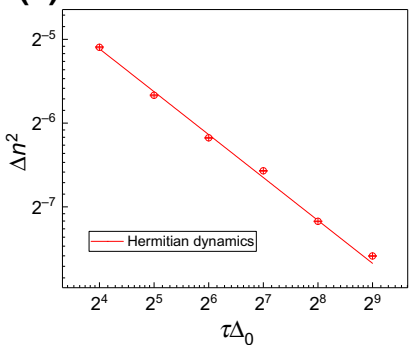

(c)

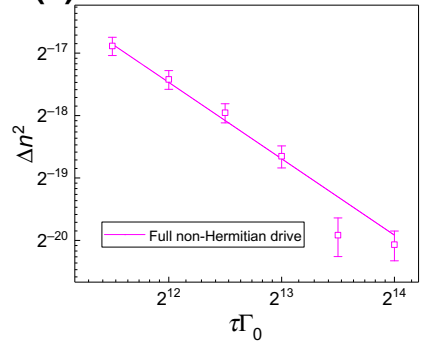

(b)

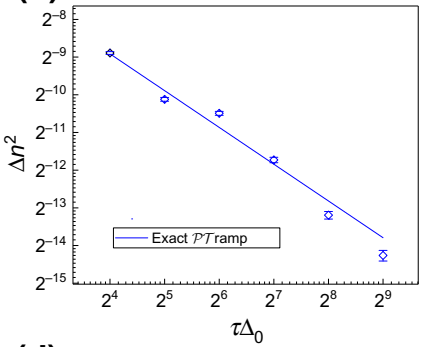

(d)

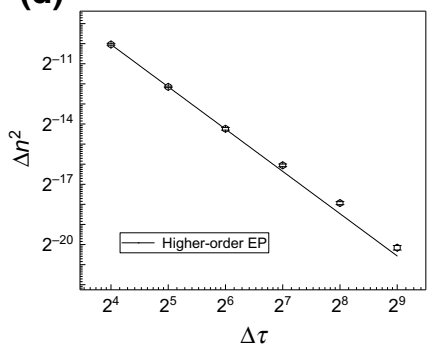

FIG. 5. The fluctuations of the defect production for different parametric ramps. The variance in the total defect densities (a) of the Hermitian case, (b) of the exact $\mathcal{P} \mathcal{T}$ case, (c) across the nonHermitian, second-order EPs, and (d) across a fourth-order EP, respectively, as functions of the ramp time $\tau$. The solid lines represent the results for the power-law fits $\Delta n^{2} \sim \tau^{\alpha}$ to the experimental data, with the fitted exponents (a) $\alpha=-0.512$ (2), (b) $\alpha=-0.97(3)$, (c) $\alpha=-1.2(1)$, and (d) $\alpha=-2.11$ (4), which correspond to the theoretical predictions (a) $\alpha=-1 / 2$, (b) $\alpha=$ -1 , (c) $\alpha=-4 / 3$, and (d) $\alpha=-2$, respectively.

near-adiabatic passage across EPs. We then go beyond the conventional defect density and generalize the scaling idea to defect fluctuations. This not only allows us to verify universal scaling in another important quantity but also enables the extraction of the correlation length exponent $v$ and dynamical exponent $z$ individually. Consequently, our work shows how the non-Hermitian Kibble-Zurek scaling can be used on a general level to experimentally gain direct information on the universality class of the EPs. The programmability of our interferometric photon network allows us to engineer both Hermitian and non-Hermitian band structures with high tunability. In particular, we demonstrate that we can also generate multiband models, which enables us to access higher-order EPs and their distinctive properties. As a key consequence, we identify a platform for realizing non-Hermitian dynamics of increasing complexity as a route toward the many-body realm with higher-dimensional time-evolution matrices.

\section{ACKNOWLEDGMENTS}

This research is supported by the National Natural Science Foundation of China (Grants No. 12025401, No. 11674056, No. 11674189, No. U1930402, and No. 11974331) and the National Research, Development and

Innovation Office (NKFIH) within the Quantum Technology National Excellence Program (Project No. 20171.2.1-NKP-2017-00001), Grant No. K119442, and by a grant from the Simons Foundation. This work was performed in part at the Aspen Center for Physics, which is supported by the National Science Foundation Grant No. PHY-1607611. Support by the Deutsche Forschungsgemeinschaft via the Leibniz Prize program SFB 1143 and cluster of excellence EXC2147 ct.qmat (Project ID 247310070 and ID 390858490) is also acknowledged. This project has received funding from the European Research Council (ERC) under the European Union's Horizon 2020 research and innovation programme (Grant Agreement No. 853443). W.Y. acknowledges support from the National Key Research and Development Program of China (Grants No. 2016YFA0301700 and No. 2017YFA0304100). K.K.W. and L.X. acknowledge support from the project funded by China Postdoctoral Science Foundation (Grants No. 2019M660016 and No. 2020M680006).

\section{APPENDIX A: THEORETICAL BACKGROUND}

As detailed in Ref. [19], we consider the time evolution of a (non-)Hermitian Hamiltonian $H(t)$. The initial state is the ground state of the starting Hamiltonian, which is always Hermitian. At time $t=0$, we start our time-dependent protocol, governed by

$$
i \partial_{t}|\Psi(t)\rangle=H(t)|\Psi(t)\rangle,
$$

with $|\Psi(t)\rangle=\otimes_{p}\left|\Psi_{p}(t)\right\rangle$ for a given mode represented by the momentum $p$, and the time dependence of $H(t)$ comes from $\Gamma(t)$ and $\Delta(t)$, always with $\Gamma(t=0)=0$.

Under the nonunitary time evolution, the norm of the wave function is not conserved, so an additional prescription for performing measurements in such states has to be given. When interpreting such dynamics as a result of dissipation in the framework of a Lindblad master equation with an additional continuous measurement, the expectation values of an operator $\mathcal{O}$ have to be evaluated as $[51,53]$

$$
\langle\mathcal{O}(t)\rangle=\frac{\langle\Psi(t)|\mathcal{O}| \Psi(t)\rangle}{\langle\Psi(t) \mid \Psi(t)\rangle}
$$

where the left state, $\langle\Psi(t)|$, is taken as the Hermitian conjugate of the time-evolved right state, $|\Psi(t)\rangle$. Since the initial condition at $t=0$ is chosen to be the ground state of a Hermitian system, the initial right and left states also satisfy this condition. In the following, we quantify the defect production via

$$
n=\frac{1}{\mathcal{N}} \sum_{p} \sigma_{z}(p), \quad \sigma_{z}(p)=\frac{\left\langle\Psi_{p}(t)\left|\sigma_{z}\right| \Psi_{p}(t)\right\rangle}{\left\langle\Psi_{p}(t) \mid \Psi_{p}(t)\right\rangle},
$$


with $\mathcal{N}$ denoting the number of considered momentum states.

The $\tau \rightarrow \infty$ limit of the momentum-resolved defect density coincides [19] with calculation of the expectation value of $\sigma_{z}$ for a given $p$ momentum state using the normalized right eigenfunction of the final Hamiltonian, corresponding to the smallest eigenvalue for the exact $\mathcal{P} \mathcal{T}$ case, or to the complex eigenvalue with the largest imaginary part otherwise. This defines $\sigma_{z}^{\mathrm{eq}}(p)$. Upon integrating this over momenta, we obtain $n_{\text {eq }}$.
The density of states of Eq. (2) with $\Delta=\Gamma=0$ is defined as $\rho(E)=(1 / \mathcal{N}) \sum_{p, \alpha= \pm} \delta\left[E-E_{\alpha}(p)\right]$ with $E_{ \pm}(p)= \pm|p|$ and $\delta(E)$ the Dirac delta function. This becomes energy, $E$, independent and takes the value quoted in the main text.

The non-Hermitian time evolution of Eq. (2) can, in principle, be solved exactly for the two-component timedependent wave function in terms of the Whittaker $\mathrm{M}$ and $\mathrm{W}$ [54], [55] functions as $\Psi(t)=\left[\Psi_{1}(t), \Psi_{2}(t)\right]^{T}$, yielding

$$
\begin{aligned}
\Psi_{1}(t)= & \frac{1}{\sqrt{t}}\left[c_{2} W\left(-\frac{1}{4}+\tau \frac{\Delta^{2}+p^{2}}{4 \Gamma}, \frac{1}{4}, t^{2} \frac{\Gamma}{\tau}\right)+c_{1} M\left(-\frac{1}{4}+\tau \frac{\Delta^{2}+p^{2}}{4 \Gamma}, \frac{1}{4}, t^{2} \frac{\Gamma}{\tau}\right)\right], \\
\Psi_{2}(t)= & \frac{\tau}{2 t^{3 / 2} \Gamma(\Delta+i p)}\left[-c_{1}\left(\Delta^{2}+p^{2}+2 \frac{\Gamma}{\tau}\right) M\left(\frac{3}{4}+\tau \frac{\Delta^{2}+p^{2}}{4 \Gamma}, \frac{1}{4}, t^{2} \frac{\Gamma}{\tau}\right)+c_{1}\left(\Delta^{2}+p^{2}\right)\right. \\
& \times M\left(-\frac{1}{4}+\tau \frac{\Delta^{2}+p^{2}}{4 \Gamma}, \frac{1}{4}, t^{2} \frac{\Gamma}{\tau}\right)+c_{2}\left(\Delta^{2}+p^{2}\right) W\left(-\frac{1}{4}+\tau \frac{\Delta^{2}+p^{2}}{4 \Gamma}, \frac{1}{4}, t^{2} \frac{\Gamma}{\tau}\right) \\
& \left.+c_{2} 4 \frac{\Gamma}{\tau} W\left(\frac{3}{4}+\tau \frac{\Delta^{2}+p^{2}}{4 \Gamma}, \frac{1}{4}, t^{2} \frac{\Gamma}{\tau}\right)\right],
\end{aligned}
$$

where the constants $c_{1,2}$ are determined from the initial condition. However, in order to obtain the $\tau$ dependence of the defect density, the scaling ansatz represents a more powerful and illuminating tool.

\section{APPENDIX B: EXPERIMENTAL REALIZATION OF (NON)UNITARY TIME EVOLUTION FOR A TWO-LEVEL SYSTEM}

In order to simulate the time evolution of these twolevel systems, we encode the basis states in the horizontal and vertical polarizations of a single photon, with $|H\rangle=$ $(1,0)^{T}$ and $|V\rangle=(0,1)^{T}$. We then extend the recipe in Ref. [56] to implement a time evolution driven by an arbitrary non-Hermitian Hamiltonian in the two-dimensional Hilbert space.

First, we numerically estimate the time-evolution operator, which, in general, is given by a $2 \times 2$ matrix $T=$ $\left(\begin{array}{ll}t_{11} & t_{12} \\ t_{21} & t_{22}\end{array}\right.$, , with complex elements $t_{i j}=\left|t_{i j}\right| e^{i \phi_{i j}}, \phi_{i j} \in \mathbb{R}$. To proceed, we define the rotation $(S)$ and phase-shift $\left(P_{ \pm}\right)$ operators:

$$
\begin{aligned}
S(\theta) & =\left(\begin{array}{cc}
\cos \theta & \sin \theta \\
-\sin \theta & \cos \theta
\end{array}\right), \quad P_{+}=\left(\begin{array}{cc}
e^{i \theta} & 0 \\
0 & 1
\end{array}\right), \\
P_{-} & =\left(\begin{array}{cc}
1 & 0 \\
0 & e^{i \theta}
\end{array}\right) .
\end{aligned}
$$

Following Ref. [56], we then decompose the matrix $T$ into a series of rotation and shift operators, as well as a loss operator $L$, which accounts for the nonunitarity of the time evolution. Specifically,

$$
\begin{aligned}
T= & P_{-}\left(\phi_{21}\right) P_{+}\left(\phi_{11}\right) S\left(\arctan \left|\frac{t_{21}}{t_{11}}\right|\right) P_{-}\left(\arg t_{22}^{\prime}\right) \\
& \times P_{+}\left(\arg t_{12}^{\prime}\right) S\left(\gamma_{1}\right) R_{\mathrm{HWP}}(\pi / 4) L S\left(\gamma_{2}\right) P_{+}\left(-\arg t_{12}^{\prime}\right),
\end{aligned}
$$

where we have

$$
\begin{aligned}
t_{11}^{\prime}= & \left|t_{11}\right| \cos \left(\arctan \left|\frac{t_{21}}{t_{11}}\right|\right)+\left|t_{21}\right| \sin \left(\arctan \left|\frac{t_{21}}{t_{11}}\right|\right), \\
t_{12}^{\prime}= & \left|t_{12}\right| \cos \left(\arctan \left|\frac{t_{21}}{t_{11}}\right|\right) e^{i\left(\phi_{21}-\phi_{11}\right)} \\
& +\left|t_{22}\right| \sin \left(\arctan \left|\frac{t_{21}}{t_{11}}\right|\right) e^{i\left(\phi_{22}-\phi_{21}\right)} \\
t_{22}^{\prime}= & -\left|t_{12}\right| \sin \left(\arctan \left|\frac{t_{21}}{t_{11}}\right|\right) e^{i\left(\phi_{12}-\phi_{11}\right)} \\
& +\left|t_{22}\right| \cos \left(\arctan \left|\frac{t_{21}}{t_{11}}\right|\right) e^{i\left(\phi_{22}-\phi_{21}\right)}
\end{aligned}
$$

Here, the loss operator is given by

$$
L=\left(\begin{array}{cc}
0 & \mu_{2} \\
\mu_{1} & 0
\end{array}\right)
$$


where $\quad \mu_{j}=\sqrt{\frac{\left|\tilde{t}_{11}\right|^{2}+\left|\tilde{t}_{22}\right|^{2}+\left|\tilde{t}_{12}\right|^{2}+(-1)^{j-1} \sqrt{q_{j}^{2}+4 p_{j}^{2}}}{2}}, \quad p_{j}=\left|\tilde{t}_{1,3-j} \tilde{t}_{3-j, 2}\right|, \quad q_{j}=\left|\tilde{t}_{11}\right|^{2}-\left|\tilde{t}_{22}\right|^{2}+(-1)^{j-1}\left|\tilde{t}_{12}\right|^{2}, \quad \tilde{t}_{11}=$ $\left|t_{11}\right| \cos \left(\arctan \left|\frac{t_{21}}{t_{11}}\right|\right)+\left|t_{21}\right| \sin \left(\arctan \left|\frac{t_{21}}{t_{11}}\right|\right), \tilde{t}_{12}=\left|t_{12}\right| \cos \left(\arctan \left|\frac{t_{21}}{t_{11}}\right|\right) e^{i\left(\varphi_{12}-\varphi_{11}\right)}+\left|t_{22}\right| \sin \left(\arctan \left|\frac{t_{21}}{t_{11}}\right|\right) e^{i\left(\varphi_{22}-\varphi_{21}\right)}$, $\tilde{t}_{22}=-\left|t_{12}\right| \sin \left(\arctan \left|\frac{t_{21}}{t_{11}}\right|\right) e^{i\left(\varphi_{12}-\varphi_{11}\right)}+\left|t_{22}\right| \cos \left(\arctan \left|\frac{t_{21}}{t_{11}}\right|\right) e^{i\left(\varphi_{22}-\varphi_{21}\right)}$.

Finally, we group the operators in Eq. (B2) into three parts,

$$
T=\underbrace{P_{-}\left(\phi_{21}\right) P_{+}\left(\phi_{11}\right) S\left(\arctan \left|\frac{t_{21}}{t_{11}}\right|\right) P_{-}\left(\arg t_{22}^{\prime}\right) P_{+}\left(\arg t_{12}^{\prime}\right) S\left(\gamma_{1}\right) R_{\mathrm{HWP}}(\pi / 4)}_{R\left(\theta_{2}, \varphi_{2}, \vartheta_{2}\right)} L \underbrace{S\left(\gamma_{2}\right) P_{+}\left(-\arg t_{12}^{\prime}\right)}_{R\left(\theta_{1}, \varphi_{1}, \vartheta_{1}\right)},
$$

thus reproducing the time-evolution operator in the main text.

Experimentally, the unitary operators $R\left(\theta_{j}, \varphi_{j}, \vartheta_{j}\right)(j=$ $1,2)$ are realized by the sandwich-type wave plates $Q\left(\vartheta_{j}\right)-H\left(\varphi_{j}\right)-Q\left(\theta_{j}\right)$ [57]. The loss operator $L$ is realized by a Mach-Zehnder interferometer involving two beam displacers (BDs) and HWPs, with the setting angles $\left(\phi_{H}, \phi_{V}\right)$ [30]. As a result of this process, for each given set of $(p, \Delta, \Gamma)$ in Eq. (2) of the main text, a set of mutually independent setting angles $\left(\theta_{1}, \varphi_{1}, \vartheta_{1}, \phi_{H}, \phi_{V}, \theta_{2}, \varphi_{2}, \vartheta_{2}\right)$ are determined. While the choice of setting angles is not unique (due to the extra degrees of freedom), our protocol chooses the analytically available one, according to Ref. [56].

In our experiment, we generate heralded single photons via type-I spontaneous parametric down-conversion, with one photon serving as the trigger and the other as the signal. The signal photon is initialized in the ground state $\left|\Psi_{p}(0)\right\rangle$ of $H_{p}$ with $\Gamma=0$ via a PBS, a QWP, and a HWP, with $p$-dependent parameters. We then send the single photon to the interferometric network as illustrated in Fig. 1.

To simulate the nonunitary dynamics, driven by a timedependent $H(t)$ up to a time $\tau$, we decompose the dynamics into different momentum sectors and directly implement the time-evolution operator $U_{p}(\tau)$. Specifically, we first numerically calculate $U_{p}(\tau)$ through

$$
U_{p}(\tau)=\prod_{k=1}^{N} e^{-i H_{p}\left(t_{k}\right) \delta t}
$$

where $t_{k}=(k-1 / 2) \delta t, \quad \delta t=\tau / N$, with $N \in \mathbb{N}$. We assume $H_{p}\left(t_{k}\right)$ to be time independent within each $k$ and take sufficiently large $N$ such that Eq. (B5) converges.

As illustrated in Fig. 1, we implement $U_{p}(\tau)$ according to

$$
U_{p}=R\left(\theta_{2}, \varphi_{2}, \vartheta_{2}\right) L\left(\phi_{H}, \phi_{V}\right) R\left(\theta_{1}, \varphi_{1}, \vartheta_{1}\right),
$$

where the rotation operator $R\left(\theta_{j}, \varphi_{j}, \vartheta_{j}\right)(j=1,2)$ is realized using a sandwich-type wave-plate set, including a
HWP at the setting angle $\varphi_{j}$ and two QWPs at $\theta_{j}$ and $\vartheta_{j}$, respectively. The polarization-dependent loss operator $L=\left(\begin{array}{cc}0 & \sin 2 \phi_{V} \\ \sin 2 \phi_{H} & 0\end{array}\right)$ is realized by a combination of two BDs and two HWPs with setting angles $\phi_{H}$ and $\phi_{V}$. The setting angles $\left\{\theta_{j}, \varphi_{j}, \vartheta_{j}, \phi_{H}, \phi_{V}\right\}$ are fixed according to the numerically calculated $U_{p}(\tau)$. We note that Eq. (B6) enables us to implement arbitrary nonunitary operators for a two-level system with different setting angles [30].

After performing the time evolution, we measure the expectation value of $\sigma_{z}$ through projective measurements. Specifically, we measure the probability of photons in the basis $\{|H\rangle,|V\rangle\}$ through a PBS and APDs. The outputs are recorded in coincidence with trigger photons. Typical measurements yield a maximum of 160000 photon counts. We then construct the momentum-resolved defect density through

$$
\sigma_{z}(p, \tau)=\frac{\left\langle\Psi_{p}(\tau)\left|\sigma_{z}\right| \Psi_{p}(\tau)\right\rangle}{\left\langle\Psi_{p}(\tau) \mid \Psi_{p}(\tau)\right\rangle}=\frac{N_{H}-N_{V}}{N_{H}+N_{V}},
$$

where $N_{H}$ and $N_{V}$ are the photon counts with horizontal and vertical polarizations, respectively.

For the variance of the total defect densities, first we construct the variance of $\sigma_{z}(p, \tau)$ through

$$
\begin{aligned}
\operatorname{Var}\left(\sigma_{z}\right)= & \left\langle\sigma_{z}^{2}\right\rangle-\left\langle\sigma_{z}\right\rangle^{2}=1^{2} \times \frac{N_{H}}{N_{H}+N_{V}}+(-1)^{2} \\
& \times \frac{N_{V}}{N_{H}+N_{V}}-\left(\frac{N_{H}}{N_{H}+N_{V}}-\frac{N_{V}}{N_{H}+N_{V}}\right)^{2} .
\end{aligned}
$$

Similarly, we can also construct the variances of $M_{y}$ and $M_{y}^{\mathrm{eq}}$. Then, we can obtain data collapse for the momentumresolved variance and the variance in the total defect densities for various cases including the Hermitian case, the exact $\mathcal{P} \mathcal{T}$ case, across non-Hermitian second-order EPs and across a fourth-order EP. The momentum-resolved variance of different cases are shown in Fig. 5, from which the universal scalings in Fig. 8 are extracted. 


\section{APPENDIX C: SPECTRUM OF $H_{p}^{(4)}$ WITH A FOURTH-ORDER EP}

The $4 \times 4$ Hamiltonian, $H_{p}^{(4)}$ in Eq. (5) can be diagonalized analytically, yielding four bands as

$$
E_{\alpha \beta}(p)=\alpha \frac{1}{2} \sqrt{2 \beta \sqrt{p^{4}-2 \Gamma^{2} p^{2}+2 p^{2} \Delta^{2}+5 \Gamma^{4}-10 \Gamma^{2} \Delta^{2}+5 \Delta^{4}+8 i p \Gamma^{3}+24 i \Gamma p \Delta^{2}}+2 p^{2}-6 \Gamma^{2}+6 \Delta^{2}},
$$

with $\alpha= \pm, \beta= \pm$. The evolution of the instantaneous spectrum is depicted in Fig. 6. For $\Gamma=\Delta$, it reduces to

$$
E_{\alpha \beta}(p)=\frac{\alpha}{\sqrt{2}} \sqrt{p^{2}+\beta \sqrt{p^{4}+32 i \Delta^{3} p}}
$$

which is dominated by the term $32 i \Delta^{3} p$ under the double square root for small momenta, thus realizing an EP4.

As mentioned in the main text, this Hamiltonian can also be rewritten in terms of the Pauli matrices of two interacting spins, denoted by $\tau_{i}$ and $\sigma_{i}$ with $i=x, y, z$. Such a minimal many-body model reads

$$
\begin{aligned}
H_{p}^{(4)}= & i p\left(\tau_{+} \sigma_{+}-\tau_{-} \sigma_{-}\right)+\Delta \sigma_{x}-i \Gamma \sigma_{y} \\
& +\tau_{+} \sigma_{-}(\Delta-\Gamma)+\tau_{-} \sigma_{+}(\Delta+\Gamma)
\end{aligned}
$$

\section{APPENDIX D: TIME EVOLUTION OF THE FOUR-LEVEL SYSTEM}

Similar to the case with two-level systems, we numerically calculate the time-evolution operator $U_{p}^{(4)}(\tau)$ at the final time $\tau$ for each $p$ sector. More explicitly, we calculate (a)

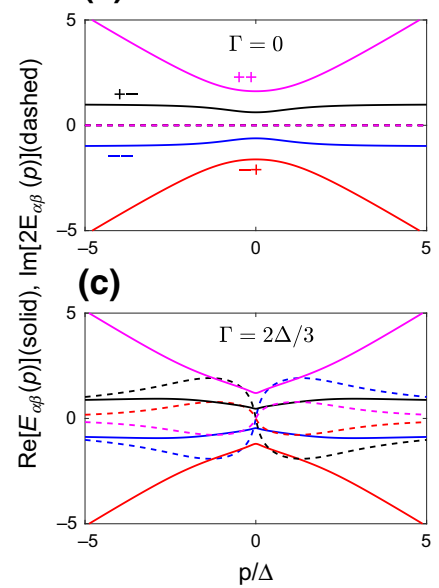

(b)

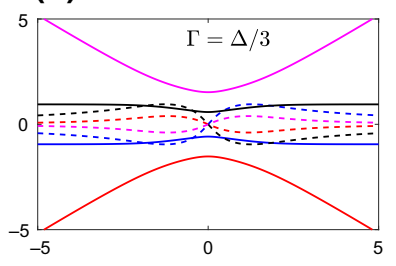

(d)

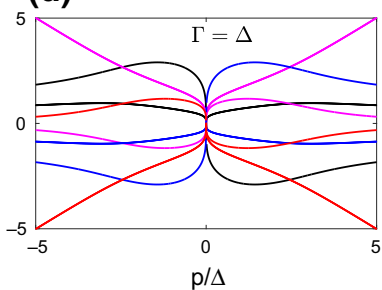

FIG. 6. The evolution of the instantaneous spectrum of $H^{(4)}$. The real and imaginary parts as a function of $\Gamma$ are shown in solid and dashed curves, respectively. The evolution starts from the Hermitian case with $\Gamma=0$ (a), through the $\mathcal{P} \mathcal{T}$-broken regions (b) and (c), and ends at $\Gamma=\Delta$ (d), where the EP4 appears at $p=0$.
$U_{p}^{(4)}(\tau)$ through $U_{p}^{(4)}=\prod_{k=1}^{N} e^{-i H_{p}^{(4)}\left(t_{k}\right) \delta t}$, for sufficiently large $N$.

To simulate the dynamics of the four-level system governed by $H^{(4)}$, we encode the four basis states in the polarizations and spatial modes of single photons, with the basis states given by $\left\{|U H\rangle=(1,0,0,0)^{T},|U V\rangle=\right.$ $\left.(0,1,0,0)^{T},|D H\rangle=(0,0,1,0)^{T},|D V\rangle=(0,0,0,1)^{T}\right\}$. Here, $|U\rangle$ and $|D\rangle$ represent, respectively, the upper and lower spatial modes of the photons [see Fig. 1(b)]. The experimental implementation of the calculated $U_{p}^{(4)}$, however, is different from that of $U_{p}$ for two-level systems, which is realized exactly by BDs and wave plates. Instead, we approximate $U_{p}^{(4)}$ with a series of modules, each consisting of two BDs and a set of wave plates. Specifically, each module features two sets of sandwiched wave plates (QWP-HWP-QWP) and a sandwiched set of BDs and wave plates (BD-HWP-BD). The QWP-HWP-QWP configuration realizes a controlled-rotation $|U\rangle\langle U| \otimes R_{U}+$ $|D\rangle\langle D| \otimes R_{D}$ and the BD-HWP-BD structure introduces the nonunitary operator

$$
L^{(4)}=\left(\begin{array}{cccc}
0 & \sin 2 \phi_{U} & 0 & 0 \\
\sin 2 \phi_{M} & 0 & 0 & -\cos 2 \phi_{M} \\
\cos 2 \phi_{M} & 0 & 0 & \sin 2 \phi_{M} \\
0 & 0 & \sin 2 \phi_{L} & 0
\end{array}\right) \text {, }
$$

where $\phi_{M}$ is the setting angle of the HWPs between two BDs.

To estimate the deviation of the implemented nonunitary time-evolution operator $U$ with respect to the ideal $U_{p}^{(4)}$, we define the distance

$$
d:=1-\frac{\left|\operatorname{Tr}\left[\tilde{U} \tilde{U}_{p}^{(4) \dagger}\right]\right|}{\sqrt{\operatorname{Tr}\left[\tilde{U} \tilde{U}^{\dagger}\right] \operatorname{Tr}\left[\tilde{U}_{p}^{(4)} \tilde{U}_{p}^{(4) \dagger}\right]}}
$$

where $\tilde{U}:=U / \operatorname{Tr}\left[U U^{\dagger}\right]$. The distance varies between 0 and 1 , with 0 indicating a perfect implementation of $U_{p}^{(4)}$. As shown in Fig. 7, the distance is already below $10^{-4}$ for the experimentally relevant parameters when we use three sets of modules. In principle, we can achieve even smaller distances by increasing the number of modules. 


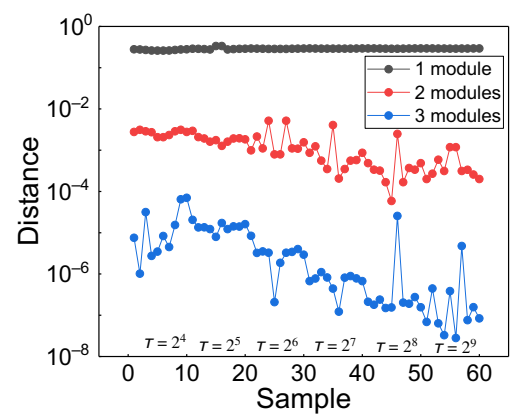

FIG. 7. The distance between the implemented nonunitary operators and the ideal ones. The calculated distance $d$ for all sampled $p$ and $\tau$, with different numbers of modules in the implementation of $U_{p}^{(4)}$. We plot the distance for all sampled $p$ with $\tau \Delta=2^{n}$ in the $x$-axis range $(10 n-40,10 n-30)$.

In order to test the Kibble-Zurek scaling around EP4 experimentally, we probe $M_{y}(p)$ via projective measurements of the time-evolved state $\left|\Psi_{p}(\tau)\right\rangle$. More specifically, we implement the transformation $O=|U H\rangle\left\langle o_{1}\right|+$ $|U V\rangle\left\langle o_{2}|+| D H\right\rangle\left\langle o_{3}|+| D V\right\rangle\left\langle o_{4}\right|$ through cascaded interferometers consisting of BDs and wave plates. Here, $\left|o_{i}\right\rangle(i=1, \ldots, 4)$ are eigenstates of $M_{y}$, with $M_{y}=$ $\sum_{i} o_{i}\left|o_{i}\right\rangle\left\langle o_{i}\right|$, where $o_{i}$ are the eigenvalues of $M_{y}$. A PBS is then used to map the basis states $\{|U H\rangle,|U V\rangle,|D H\rangle,|D V\rangle\}$ to four distinct spatial modes, where photons are collected by four APDs. It follows that $M_{y}(p)=\sum_{i=1}^{4} o_{i} P_{i}$, where $P_{i}$ is the photon count from the corresponding APD. We note (a)

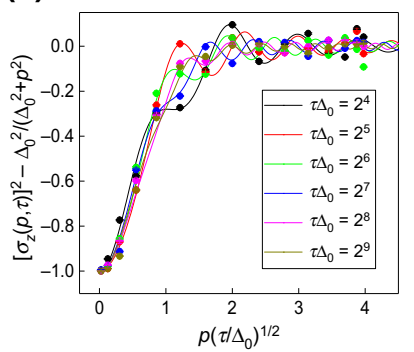

(c)

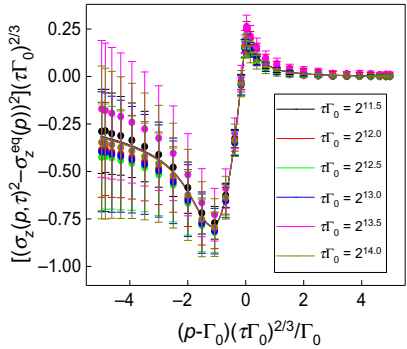

(b)

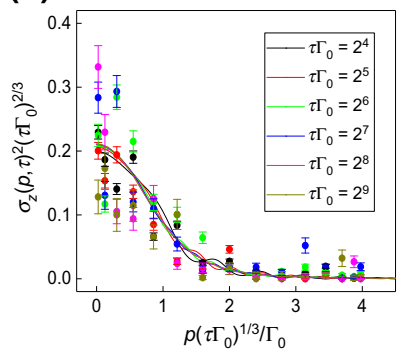

(d)

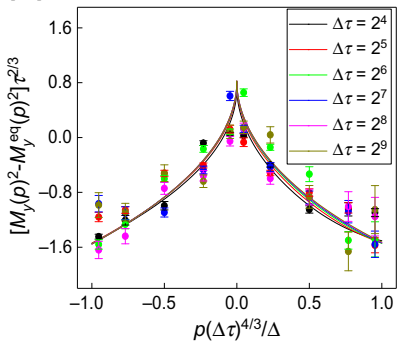

FIG. 8. The fluctuations of the defect production for different parametric ramps. The data collapse for the momentum-resolved variance (a) for the Hermitian case, (b) for the exact $\mathcal{P} \mathcal{T}$ case, (c) across the non-Hermitian second-order EPs, and (d) across a fourth-order EP. that $M_{y}^{\mathrm{eq}}$ is measured in a similar fashion, by performing projective measurements directly on the state $\left|\varphi_{p}\right\rangle$.

\section{APPENDIX E: ERROR ANALYSIS}

The difference between the experimental data and the theoretical predictions is mostly caused by the inaccuracy of the wave-plate parameters, as well as the dephasing of BDs in the cascaded interferometric network. Since both wave plates and BDs are tuned independently in different $p$ sectors, experimental errors in different $p$ sectors are uncorrelated.

For the data shown in Fig. 2(a), the unitary time evolution is realized by three HWPs and without any interferometers (hence no BD). In contrast, for the data shown in Figs. 2(b), 3(a), and 4(a), the nonunitary time evolutions therein require more wave plates as well as BDs. This is the reason for the more apparent experimental imperfections in the case of nonunitary time evolutions. However, since the imperfections in each $p$ are independent (see the above discussion) and the data shown in Figs. 2(c), 3(b), and 4(b) are obtained by integrating over $p$, the differences between the experimental data and the theoretical predictions are smaller for the total defect densities.

\section{APPENDIX F: SCALING OF DEFECT FLUCTUATIONS}

For the sake of simplicity, we consider a second-order EP. Using the fact that the wave function factorizes for different momenta and the expectation values can thus be taken independently for different momenta, this is rewritten as

$$
\Delta n^{2}=\mathcal{N}^{-1} \sum_{p}\left\{\left[\sigma_{z}(p, \tau)\right]^{2}-\left[\sigma_{z}^{\mathrm{eq}}(p)\right]^{2}\right\}
$$

Let us first discuss the case with $\sigma_{z}^{\mathrm{eq}}(p)=0$ for all $p$, which is the case for the exact $\mathcal{P} \mathcal{T}$ ramp. Using the scaling form, we obtain

$$
\begin{aligned}
\Delta n^{2}= & \int \frac{d p}{2 \pi}\left[\sigma_{z}(p, \tau)\right]^{2} \\
= & \frac{1}{2 \pi} \int d p \frac{1}{\tau^{2 z \nu /(z v+1)}} f^{2}\left(p^{z} \tau^{z v /(z v+1)}\right) \\
& \times \sim \tau^{-v(d+2 z) /(z v+1)} .
\end{aligned}
$$

For the other case with a finite $\sigma_{z}^{\mathrm{eq}}(p)$, we can rewrite $\left[\sigma_{z}(p, \tau)\right]^{2}-\left[\sigma_{z}^{\mathrm{eq}}(p)\right]^{2}=\left[\sigma_{z}(p, \tau)-\sigma_{z}^{\mathrm{eq}}(p)\right]\left[\sigma_{z}(p, \tau)+\right.$ $\left.\sigma_{z}^{\mathrm{eq}}(p)\right] \approx\left[\sigma_{z}(p, \tau)-\sigma_{z}^{\mathrm{eq}}(p)\right] 2 \sigma_{z}^{\mathrm{eq}}(p)$, where $\mid \sigma_{z}(p, \tau)-$ $\sigma_{z}^{\text {eq }}(p)|\ll| \sigma_{z}^{\text {eq }}(p) \mid$ close to the adiabatic, $\tau \rightarrow \infty$ limit. From the calculations for all EPs, it turns out that 
$\sigma_{z}^{\mathrm{eq}}(p) \sim|p|^{z}$, which allows us to write

$$
\begin{aligned}
\Delta n^{2} & \approx \int \frac{d p}{2 \pi}\left[\sigma_{z}(p, \tau)-\sigma_{z}^{\mathrm{eq}}(p)\right] 2 \sigma_{z}^{\mathrm{eq}}(p) \\
& \sim \int \frac{d p}{2 \pi} \frac{|p|^{z}}{\tau^{z v /(z v+1)}} f\left(p^{z} \tau^{z v /(z v+1)}\right) \\
& \sim \tau^{-v(d+2 z) /(z v+1)}
\end{aligned}
$$

recovering the previous expression.

For a conventional Hermitian critical point, the prefactors $\tau^{z v /(z v+1)}$ as well as the additional momentumdependent terms in the integrals are missing; thus we recover the $\Delta n^{2} \sim \tau^{-v d /(z v+1)}$ scaling for that case.

[1] R. El-Ganainy, K. G. Makris, M. Khajavikhan, Z. H. Musslimani, S. Rotter, and D. N. Christodoulides, NonHermitian physics and PT symmetry, Nat. Phys. 14, 11 (2018).

[2] J. M. Zeuner, M. C. Rechtsman, Y. Plotnik, Y. Lumer, S. Nolte, M. S. Rudner, M. Segev, and A. Szameit, Observation of a Topological Transition in the Bulk of a NonHermitian System, Phys. Rev. Lett. 115, 040402 (2015).

[3] L. Xiao, X. Zhan, Z. Bian, K. Wang, X. Zhang, X. Wang, J. Li, K. Mochizuki, D. Kim, N. Kawakami, W. Yi, H. Obuse, B. C. Sanders, and P. Xue, Observation of topological edge states in parity-time-symmetric quantum walks, Nat. Phys. 13, 1117 (2017).

[4] X. Zhan, L. Xiao, Z. Bian, K. Wang, X. Qiu, B. C. Sanders, W. Yi, and P. Xue, Detecting Topological Invariants in Nonunitary Discrete-Time Quantum Walks, Phys. Rev. Lett. 119, 130501 (2017).

[5] J. Li, A. K. Harter, J. Liu, L. de Melo, Y. N. Joglekar, and L. Luo, Observation of parity-time symmetry breaking transitions in a dissipative Floquet system of ultracold atoms, Nat. Commun. 10, 855 (2019).

[6] S. Lapp, J. Ang'ong'a, F. A. An, and B. Gadway, Engineering tunable local loss in a synthetic lattice of momentum states, New J. Phys. 21, 045006 (2019).

[7] M. Lebrat, S. Häusler, P. Fabritius, D. Husmann, L. Corman, and T. Esslinger, Quantized Conductance through a Spin-Selective Atomic Point Contact, Phys. Rev. Lett. 123, 193605 (2019).

[8] L. Corman, P. Fabritius, S. Häusler, J. Mohan, L. H. Dogra, D. Husmann, M. Lebrat, and T. Esslinger, Quantized conductance through a dissipative atomic point contact, Phys. Rev. A 100, 053605 (2019).

[9] Y. Takasu, T. Yagami, Y. Ashida, R. Hamazaki, Y. Kuno, and Y. Takahashi, PT-symmetric non-Hermitian quantum many-body system using ultracold atoms in an optical lattice with controlled dissipation, arXiv:2004.05734.

[10] C. M. Bender, B. K. Berntson, D. Parker, and E. Samuel, Observation of $\mathcal{P} \mathcal{T}$ phase transition in a simple mechanical system, Am. J. Phys. 81, 173 (2013).

[11] E. Rivet, A. Brandstotter, K. G. Makris, H. Lissek, S. Rotter, and R. Fleury, Constant-pressure sound waves in nonHermitian disordered media, Nat. Phys. 14, 942 (2018).
[12] T. Yoshida and Y. Hatsugai, Exceptional rings protected by emergent symmetry for mechanical systems, Phys. Rev. B 100, 054109 (2019).

[13] T. Stehmann, W. D. Heiss, and F. G. Scholtz, Observation of exceptional points in electronic circuits, J. Phys. A: Math. Gen. 37, 7813 (2004).

[14] J. Schindler, A. Li, M. C. Zheng, F. M. Ellis, and T. Kottos, Experimental study of active LRC circuits with $\mathcal{P} \mathcal{T}$-symmetries, Phys. Rev. A 84, 040101(R) (2011).

[15] R. Fleury, D. Sounas, and A. Alú, An invisible acoustic sensor based on parity-time symmetry, Nat. Commun. 6, 1 (2015).

[16] M. Ezawa, Electric-circuit simulation of the Schrödinger equation and non-Hermitian quantum walks, Phys. Rev. B 100, 165419 (2019).

[17] T. Yoshida, T. Mizoguchi, and Y. Hatsugai, Mirror skin effect and its electric circuit simulation, arXiv preprint arXiv:1912.12022 (2019).

[18] W. D. Heiss, The physics of exceptional points, J. Phys. A: Math. Theor. 45, 444016 (2012).

[19] B. Dóra, M. Heyl, and R. Moessner, The Kibble-Zurek mechanism at exceptional points, Nat. Commun. 10, 2254 (2019).

[20] H. Hodaei, A. U. Hassan, S. Wittek, H. Garcia-Gracia, R. El-Ganainy, D. N. Christodoulides, and M. Khajavikhan, Enhanced sensitivity at higher-order exceptional points, Nature 548, 187 (2017).

[21] Ş. Özdemir, S. Rotter, F. Nori, and L. Yang, Parity-time symmetry and exceptional points in photonics, Nat. Mater. 18, 783 (2019).

[22] R. Hanai and P. B. Littlewood, Critical fluctuations at a many-body exceptional point, arXiv preprint arXiv:1908. 03243 (2019).

[23] C. M. Bender and S. Boettcher, Real Spectra in NonHermitian Hamiltonians Having $\mathcal{P} \mathcal{T}$ Symmetry, Phys. Rev. Lett. 80, 5243 (1998).

[24] B. T. Torosov and N. V. Vitanov, Pseudo-Hermitian Landau-Zener-Stückelberg-Majorana model, Phys. Rev. A 96, 013845 (2017).

[25] B. T. Torosov, G. D. Valle, and S. Longhi, Non-Hermitian shortcut to adiabaticity, Phys. Rev. A 87, 052502 (2013).

[26] S. Yin, G.-Y. Huang, C.-Y. Lo, and P. Chen, Kibble-Zurek Scaling in the Yang-Lee Edge Singularity, Phys. Rev. Lett. 118, 065701 (2017).

[27] Y. Ashida, S. Furukawa, and M. Ueda, Parity-timesymmetric quantum critical phenomena, Nat. Commun. 8, 15791 (2017).

[28] S. Sachdev, Quantum Phase Transitions (Cambridge University Press, Cambridge, 1999).

[29] K. Kawabata, Y. Ashida, and M. Ueda, Information Retrieval and Criticality in Parity-Time-Symmetric Systems, Phys. Rev. Lett. 119, 190401 (2017).

[30] L. Xiao, K. Wang, X. Zhan, Z. Bian, K. Kawabata, M. Ueda, W. Yi, and P. Xue, Observation of Critical Phenomena in Parity-Time-Symmetric Quantum Dynamics, Phys. Rev. Lett. 123, 230401 (2019).

[31] T. W. B. Kibble, Topology of cosmic domains and strings, J. Phys. A 9, 1387 (1976).

[32] W. H. Zurek, Cosmological experiments in superfluid helium?, Nature 317, 505 (1985). 
[33] A. Polkovnikov, K. Sengupta, A. Silva, and M. Vengalattore, Colloquium: Nonequilibrium dynamics of closed interacting quantum systems, Rev. Mod. Phys. 83, 863 (2011).

[34] P. Hedvall and J. Larson, Dynamics of non-equilibrium steady state quantum phase transitions, arXiv preprint arXiv:1712.01560 (2017).

[35] X.-Y. Xu, Y.-J. Han, K. Sun, J.-S. Xu, J.-S. Tang, C.-F. Li, and G.-C. Guo, Quantum Simulation of Landau-Zener Model Dynamics Supporting the Kibble-Zurek Mechanism, Phys. Rev. Lett. 112, 035701 (2014).

[36] M. Gong, X. Wen, G. Sun, D. Zhang, D. Lan, Y. Zhou, Y. Fan, Y. Liu, X. Tan, H. Yu, Y. Yu, S.-L. Zhu, S. Han, and P. $\mathrm{Wu}$, Simulating the Kibble-Zurek mechanism of the ising model with a superconducting qubit system, Sci. Rep. 6, 22667 (2016)

[37] M. Anquez, B. Robbins, H. Bharath, M. Boguslawski, T. Hoang, and M. Chapman, Quantum Kibble-Zurek Mechanism in a Spin-1 Bose-Einstein Condensate, Phys. Rev. Lett. 116, 155301 (2016).

[38] G. Labeyrie and R. Kaiser, Kibble-Zurek Mechanism in the Self-Organization of a Cold Atomic Cloud, Phys. Rev. Lett. 117, 275701 (2016).

[39] A. Keesling, A. Omran, H. Levine, H. Bernien, H. Pichler, S. Choi, R. Samajdar, S. Schwartz, P. Silvi, S. Sachdev, P. Zoller, M. Endres, M. Greiner, V. Vuletić, and M. D. Lukin, Quantum Kibble-Zurek mechanism and critical dynamics on a programmable Rydberg simulator, Nature 568, 207 (2019).

[40] R. Landauer, The noise is the signal, Nature 392, 658 (1998).

[41] J. Doppler, A. A. Mailybaev, J. Bohm, U. Kuhl, A. Girschik, F. Libisch, T. J. Milburn, P. Rabl, N. Moiseyev, and S. Rotter, Dynamically encircling an exceptional point for asymmetric mode switching, Nature 537, 76 (2016).

[42] S. Longhi, Convective and absolute $\mathcal{P} \mathcal{T}$-symmetry breaking in tight-binding lattices, Phys. Rev. A 88, 052102 (2013).

[43] T. E. Lee, Anomalous Edge State in a Non-Hermitian Lattice, Phys. Rev. Lett. 116, 133903 (2016).
[44] T. Gao, E. Estrecho, K. Y. Bliokh, T. C. H. Liew, M. D. Fraser, S. Brodbeck, M. Kamp, C. Schneider, S. Höfling, Y. Yamamoto, F. Nori, and Y. S. Kivshar et al., Observation of non-Hermitian degeneracies in a chaotic exciton-polariton billiard, Nature 526, 554 (2015).

[45] see Appendix for details.

[46] W. D. Heiss, M. Müller, and I. Rotter, Collectivity, phase transitions, and exceptional points in open quantum systems, Phys. Rev. E 58, 2894 (1998).

[47] T. J. Milburn, J. Doppler, C. A. Holmes, S. Portolan, S. Rotter, and P. Rabl, General description of quasiadiabatic dynamical phenomena near exceptional points, Phys. Rev. A 92, 052124 (2015).

[48] H. Xu, D. Mason, L. Jiang, and J. G. E Harris, Topological energy transfer in an optomechanical system with an exceptional point, Nature 537, 80 (2016).

[49] L. Saminadayar, D. C. Glattli, Y. Jin, and B. Etienne, Observation of the $e / 3$ Fractionally Charged Laughlin Quasiparticle, Phys. Rev. Lett. 79, 2526 (1997).

[50] J.-M. Cui, F. J. Gómez-Ruiz, Y.-F. Huang, C.-F. Li, G.-C. Guo, and A. del Campo, Experimentally testing quantum critical dynamics beyond the Kibble-Zurek mechanism, Commun. Phys. 3, 44 (2020).

[51] Y. Ashida and M. Ueda, Full-Counting Many-Particle Dynamics: Nonlocal and Chiral Propagation of Correlations, Phys. Rev. Lett. 120, 185301 (2018).

[52] E. M. Graefe, H. J. Korsch, and A. E. Niederle, Mean-Field Dynamics of a Non-Hermitian Bose-Hubbard Dimer, Phys. Rev. Lett. 101, 150408 (2008).

[53] H. Carmichael, An Open Systems Approach to Quantum Optics (Springer-Verlag, Berlin, 1993).

[54] I. Gradshteyn and I. Ryzhik, Table of Integrals, Series, and Products (Academic Press, New York, 2007).

[55] V. M. Akulin and W. P. Schleich, Landau-Zener transition to a decaying level, Phys. Rev. A 46, 4110 (1992).

[56] N. Tischler, C. Rockstuhl, and K. Słowik, Quantum Optical Realization of Arbitrary Linear Transformations Allowing for Loss and Gain, Phys. Rev. X 8, 021017 (2018).

[57] B. Neethi Simon, C. M. Chandrashekar, and Sudhavathani Simon, Hamilton's turns as a visual tool kit for designing single-qubit unitary gates, Phys. Rev. A 85, 022323 (2012). 